\title{
Characterization of an AIN buffer layer and a thick-GaN layer grown on sapphire substrate by MOCVD
}

\author{
S. Çörekçi • M. K. Öztürk • A. Bengi • \\ M. Çakmak · S. Özçelik • E. Özbay
}

Received: 26 March 2010/Accepted: 4 October 2010/Published online: 23 October 2010

(C) Springer Science+Business Media, LLC 2010

\begin{abstract}
An AlN buffer layer and a thick-GaN layer for high-electron-mobility transistors (HEMTs) were grown on sapphire substrate by metal-organic chemical vapor deposition (MOCVD). The structural and morphological properties of the layers were investigated by high resolution X-ray diffraction (HRXRD) and atomic force microscopy (AFM) techniques. The optical quality of the thick-GaN layer was also evaluated in detail by a photoluminescence (PL) measurement. It was found that the AlN buffer layer possesses high crystal quality and an atomically flat surface with a root-mean-square (rms) roughness of $0.16 \mathrm{~nm}$. The screw- and edge-type dislocation densities of the thick-GaN layer were determined as $5.4 \times 10^{7}$ and $5.0 \times 10^{9} \mathrm{~cm}^{-2}$ by means of the mosaic crystal model, respectively. It was observed that the $\mathrm{GaN}$ layer has a smooth surface with an rms of $0.84 \mathrm{~nm}$. Furthermore, the dark spot density of the GaN surface was estimated as $6.5 \times 10^{8} \mathrm{~cm}^{-2}$ over a scan area of $4 \mu \mathrm{m}^{2}$.
\end{abstract}

\section{Introduction}

GaN-based high-electron-mobility transistors (HEMTs) have great potential for high power, high temperature, and

S. Çörekçi (凹)

Physics Department, Kırklareli University, 39160 Kirklareli,

Turkey

e-mail: scorekci@kirklareli.edu.tr

M. K. Öztürk · A. Bengi · M. Çakmak · S. Özçelik

Physics Department, Gazi University, 06500 Ankara, Turkey

E. Özbay

Nanotechnology Research Center, Bilkent University,

06800 Ankara, Turkey high frequency applications $[1,2]$. The thick-GaN layers $(\sim 2000 \mathrm{~nm})$ in HEMTs are generally grown on sapphire substrates due to their lower cost, higher temperature stability, and mature growth technology [2]. Since GaN and sapphire materials have different lattice parameters and thermal expansion coefficients, the large lattice mismatch and thermal mismatch between these materials lead to high dislocation densities of $10^{7}-10^{11} \mathrm{~cm}^{-2}$ in a GaN epilayer grown on sapphire substrate [3-6]. Dislocations in the GaN layers are deleterious to device performance due to electron scattering and current leakage paths $[5,6]$. Thus, the preparation of good quality thick-GaN layers is quite important for HEMTs. One approach to avoid a large lattice mismatch between $\mathrm{GaN}$ and sapphire materials, is to grow a thin low-temperature (LT) GaN layer or AlN nucleation layer prior to the growth of the thick-GaN layer $[7,8]$. In addition, it has already been reported that the crystalline quality of $\mathrm{GaN}$ epilayers that are grown on high temperature AlN buffer layers was considerably improved $[9,10]$. Although there are several reports about the effects of an AlN buffer layer on GaN epilayers, there is still a lack of detailed research on a thick-GaN layer achieved by using an AlN buffer layer.

We have previously reported the growth of high quality $\mathrm{AlGaN} / \mathrm{GaN}$ heterostructures with a high-temperature AlN buffer layer on sapphire substrates by metal-organic chemical vapor deposition (MOCVD) for HEMTs [11]. In this study, we investigated the structural quality and surface morphology of an AlN buffer layer and a thick-GaN layer by high resolution X-ray diffraction (HRXRD) and atomic force microscopy (AFM). We calculated the threading dislocation (TD) densities of the GaN layer by means of the mosaic crystal model. We also evaluated the room temperature photoluminescence (PL) spectra of the $\mathrm{GaN}$ layer. 


\section{Experiments}

The samples that were used in the present study were grown on $c$-plane sapphire substrates by low-pressure MOCVD. Hydrogen $\left(\mathrm{H}_{2}\right)$ was used as the carrier gas, and trimethylgallium (TMGa), trimethylaluminum (TMAl), and ammonia $\left(\mathrm{NH}_{3}\right)$ were used as the $\mathrm{Ga}, \mathrm{Al}$, and $\mathrm{N}$ sources, respectively. Prior to epitaxial growth, sapphire substrates were annealed at $1100{ }^{\circ} \mathrm{C}$ for $10 \mathrm{~min}$ in order to remove surface contamination. As shown in Fig. 1, an AlN buffer/sapphire template and a thick-GaN layer grown on an AlN buffer/sapphire template were labeled as samples A and B. For sample A, a 15-nm-thick AlN nucleation layer was deposited at $840{ }^{\circ} \mathrm{C}$. Subsequently, the reactor temperature was ramped to $1150{ }^{\circ} \mathrm{C}$ and a 500 -nm-thick AlN buffer layer was grown. The 500-nm-thick AlN buffer layer was grown at a reactor pressure of $25 \mathrm{mbar}$, a growth temperature of $1150{ }^{\circ} \mathrm{C}$, and a growth rate of $0.5 \mu \mathrm{m} / \mathrm{h}$. For sample B, first a 15 -nm-thick AlN nucleation layer was deposited at $840{ }^{\circ} \mathrm{C}$. Subsequently, the reactor temperature was ramped to $1150{ }^{\circ} \mathrm{C}$ and a 500-nm-thick AlN buffer layer was grown, followed by a 2-min growth interruption in order to reach growth conditions for GaN. Afterwards, the 2000-nm-thick GaN layer was grown at a reactor pressure of 200 mbar, a growth temperature of $1070{ }^{\circ} \mathrm{C}$, and a growth rate of $2.0 \mu \mathrm{m} / \mathrm{h}$.

The structural quality, surface morphology, and optical properties of the samples were characterized by HRXRD, AFM, and PL measurements. HRXRD measurements were performed by a Bruker D8-Discover high resolution diffractometer $\left(0.0044^{\circ}\right.$ for the Si calibration sample) by using $\mathrm{Cu} \mathrm{K} \alpha_{1}(0.154 \mathrm{~nm})$ radiation, a prodded mirror, and a 4-bounce $\mathrm{Ge}(220)$ symmetric monochromator. For asymmetric reflections, the system has four circles consist of omega, $2 \times$ theta, chi, and phi axes. AFM images of the samples were recorded using an Omicron VT STM/AFM system. The root-mean-square (rms) roughness values of the samples were processed from the surface topography

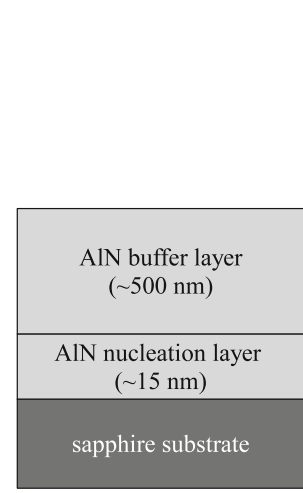

(a)

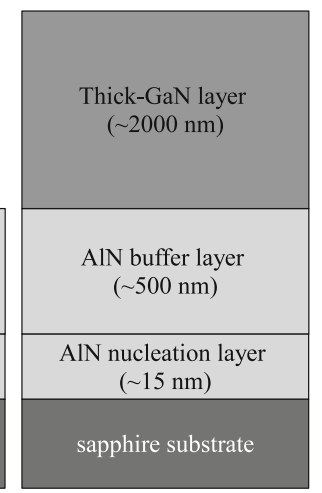

(b)
Fig. 1 Schematic structures of the samples $\mathbf{a}$ A and $\mathbf{b}$ B using Scala Pro software. Optical measurements were performed at room temperature using a Horiba Jobin-Yvon Fluorolog3 conventional photoluminescence system with a $325 \mathrm{~nm}$ line of a $50 \mathrm{~mW} \mathrm{He}-\mathrm{Cd}$ laser as an excitation source.

\section{Results and discussion}

XRD symmetric (002) and asymmetric (102) scans are reliable techniques for characterizing the structural quality of the nitride layers [2]. Figure 2 shows the (002) $\omega-2 \theta$ scans of samples A and B, in which the results confirm the nominal structures illustrated in Fig. 1 except for sapphire substrates. The peak in sample A is a Bragg reflection from the (002) plane of the AlN layer. The two main peaks in sample B are Bragg reflections from the (002) planes of the $\mathrm{GaN}$ and AlN layers, respectively. As can be seen in Fig. 2, the AlN peak of sample B is significantly broader than that of sample A and the peak positions for both samples are different. These results are related to the strain state, defective structure, and imperfect stoichiometry of the AlN layers. More detailed information on the structural quality of the samples was obtained by (002) and (102) X-ray $\omega$-scans. From the $\omega$-scans of the AlN layers (not shown here), the full-width at half-maximums (FWHMs) of the (002) and (102) reflections were determined as $0.012^{\circ}$, $0.247^{\circ}$ for sample $A$, and $0.044^{\circ}, 0.347^{\circ}$ for sample B as listed in Table 1. The (102) FWHM value for both of the samples was notably higher than that of the (002) FWHM. These results are in good agreement with the previously reported results about high quality AlN layers [8, 12-14]. For example, Ha et al. [13] investigated a 1000-nm-thick AlN epilayer grown on sapphire substrate by MOCVD and

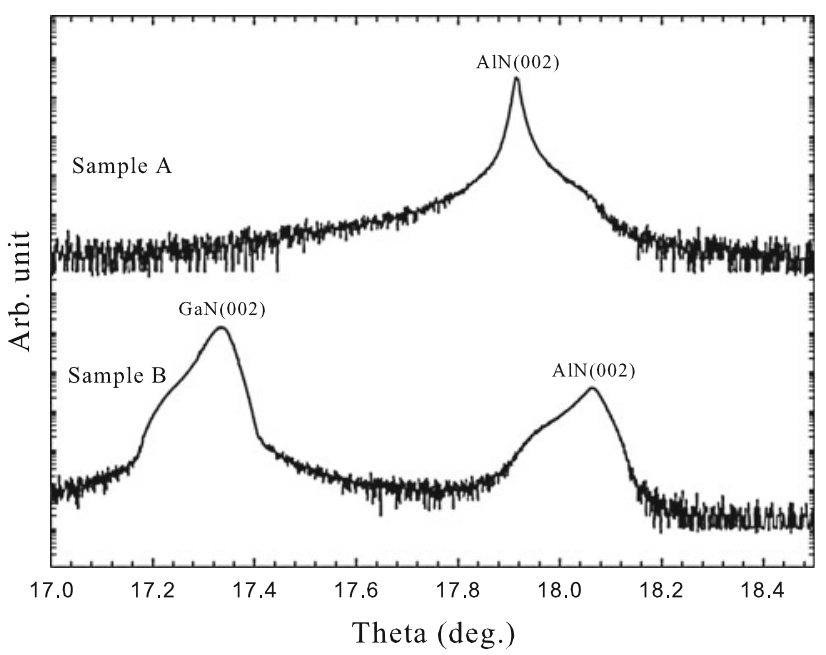

Fig. 2 High-resolution Bragg reflections near the (002) reflection of the samples 
Table 1 The structural parameters of the samples

\begin{tabular}{|c|c|c|c|c|c|c|c|}
\hline \multirow[t]{2}{*}{ Sample } & \multirow[t]{2}{*}{ Layers } & \multicolumn{2}{|c|}{ FWHM $\left(^{\circ}\right)$} & \multicolumn{2}{|c|}{$\begin{array}{l}\text { Lattice } \\
\text { parameters }(\AA)\end{array}$} & \multicolumn{2}{|l|}{ Strain } \\
\hline & & $(002)$ & (102) & $a$ & $c$ & $\varepsilon_{\mathrm{a}}$ & $\varepsilon_{\mathrm{c}}$ \\
\hline Sample A & AlN & 0.012 & 0.247 & 3.6448 & 4.9917 & 0.1714 & 0.0025 \\
\hline \multirow[t]{2}{*}{ Sample B } & $\mathrm{AlN}$ & 0.044 & 0.347 & 3.1494 & 4.8768 & 0.0122 & -0.0206 \\
\hline & $\mathrm{GaN}$ & 0.043 & 0.207 & 3.1885 & 5.1779 & -0.0004 & -0.0015 \\
\hline
\end{tabular}

found that the AlN epilayer has FWHMs of $0.020^{\circ}, 0.288^{\circ}$ for the (002) and (102) reflections, respectively. Similarly, Katagiri et al. [14] reported that the (002) and (102) FWHM values of an AlN layer on flat AlN/sapphire template are $0.109^{\circ}, 0.259^{\circ}$.

The broadening of the $\omega$-scans is mainly related to the mosaicity, grain size, and microstrain $[15,16]$. The mosaicity is a measure of the grain misorientation and is associated with the TDs in the epitaxial films [15]. As is well known, the TDs are of three types in a GaN epilayer: screw (c-type) with Burgers vector $b=\langle 0001\rangle$, edge ( $a$-type) with Burgers vector $b=1 / 3\langle 11 \overline{2} 0\rangle$, and mixed ( $c+a$-type) with Burgers vector $b=1 / 3\langle 11 \overline{2} 3\rangle$. The screw TDs distorts all the $\{h k i l\}$ planes with 1 non-zero, while edge TDs only distorts the $\{h k i l\}$ planes with $h$ or $k$ non-zero [17]. For this reason, the screw or mixed TDs make a contribution to the broadening of the (002) $\omega$-scan, while all the TDs contribute to the broadening of the (102) $\omega$-scan [8]. Within this framework, the dramatic broadening of the (102) $\omega$-scans compared to the (002) $\omega$-scans shows that the AlN layers in the samples have a large edge TD density. On the other hand, the crystal quality of the AlN in sample B is lower than that of sample A in spite of having the same growth conditions of the AlN layers for both of the samples. The control of the growth temperature, which might change the flow rates during growth, is rather critical. The high growth temperature $\left(1150{ }^{\circ} \mathrm{C}\right)$ of the AlN buffer layer probably leads to rough surfaces. Therefore, the low crystalline quality of the AlN in sample B may be attributed to the defective microstructure of GaN/AlN interface. In addition, the large thermal strain between AlN and GaN layers becomes important by exhibiting a compressive behavior in -0.0010 value calculated by $\varepsilon=$ $\left(\alpha_{\mathrm{AIN}}-\alpha_{\mathrm{GaN}}\right) \Delta T$, where here $\alpha_{\mathrm{AIN}}, \alpha_{\mathrm{GaN}}$ are the thermal expansion constants $5.59 \times 10^{-6}, 6.90 \times 10^{-6}$ [18-20] and $\Delta T$ is the temperature difference derived from the postgrowth cooling from the growth temperature to room temperature for the layers. This value shows that there is a little plastic deformation during cooling.

Figure 3 shows the X-ray $\omega$-scans that were obtained from the (002) and (102) planes of the thick-GaN layer that was grown using an AlN buffer (sample B). The $\omega$-scans were fitted into a curve determined by a Pseudo-Voigt function. From the fitted curves, the FWHM values of the (002) and (102) reflections were obtained as $0.043^{\circ}$ and $0.207^{\circ}$. Generally, the typical (002) and (102) FWHMs of $\mathrm{GaN}$ are around $0.069^{\circ}-0.083^{\circ}$ and $0.083^{\circ}-0.097^{\circ}[8,21]$. The (002) FWHM value of the $\mathrm{GaN}$ is better than that of typical GaN, while the (102) FWHM value is higher. At the same time, (102) FWHM of the GaN is lower than that of (102) of the AlN. This result shows that the edge-type dislocation density of the GaN is lower than that of AlN.

GaN epitaxial layers with high dislocation density are often described by the mosaic model [22]. As seen in Fig. 4, a mosaic layer consists of single crystalline blocks with lateral and vertical coherence lengths. The out-ofplane rotation of these blocks, which are perpendicular to the surface normal, is a tilt, and the in-plane rotation around the surface normal is a twist [22]. In a mosaic GaN epilayer, XRD peaks are broadened by several different features such as tilt, twist, limited crystallite size, and microstrain [16]. To evaluate the mosaicity of the thick$\mathrm{GaN}$ layer, symmetric $\omega$-scans and azimuthal $\phi$-scans were performed. The FWHM values of the $(00 \ell)(\ell=$ 2,4 , and 6) reflections were determined by fitting the $\omega$-scan curves to the Pseudo-Voigt function and they were used for the Williamson-Hall (WH) plot, which gives a
Fig. 3 The (002) and (102) $\omega$-scans of a thick-GaN layer and the corresponding Pseudo-Voigt fits
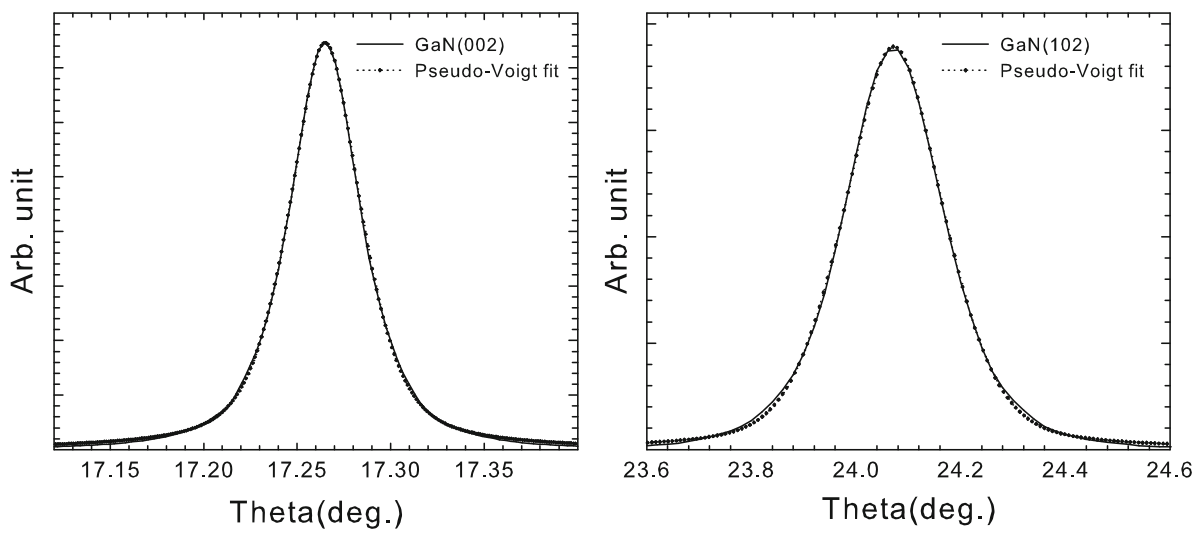


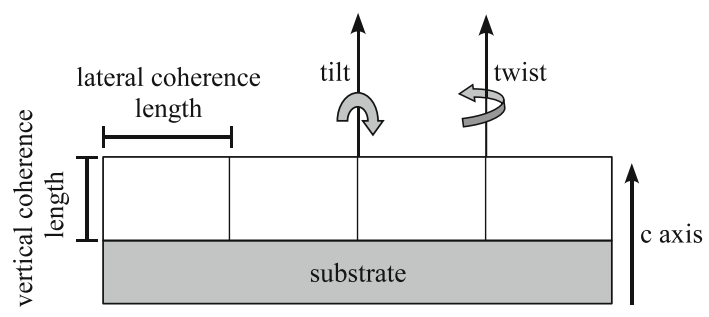

Fig. 4 Illustration of a mosaic layer

measure of the tilt [16]. Figure 5a shows the variation of $\mathrm{FWHM} \times \sin (\theta) / \lambda$ versus $\sin (\theta) / \lambda$ as a function of reflection order, where $\theta$ is the Bragg reflection angle, $\lambda$ is the wavelength of the $\mathrm{Cu} \mathrm{K} \alpha_{1}$. The tilt angle of the $\mathrm{GaN}$ layer is obtained as $0.046^{\circ}$ from the slope of the linear dependence in Fig. 5a. Figure 5b shows the FWHMs of the $\omega$ - and $\phi$-scans as a function of the $\chi$ angle for GaN. As seen in Fig. 5b, the FWHMs of $\omega$-scans in turn increase, while the FWHMs of the $\phi$-scans decrease with the increment in $\chi$ angle. Moreover, they become closer when the (121) reflection yields at 78.6 in the $\chi$ angle. The FWHM value of the $\omega$ - and $\phi$-scans in the $\chi=78.6$ gives a measure of the mean twist [23]. The twist angle for the $\mathrm{GaN}$ layer is obtained as $0.274^{\circ}$.

The screw and edge TD densities in GaN films are related with mosaic tilt and twist values, respectively [24]. To determine dislocation densities in thick-GaN layer, we used a technique proposed by Metzger et al. [25]. The density of screw TDs can be obtained using the following equation:

$\rho_{\mathrm{s}}=\frac{\alpha_{\Omega}^{2}}{4.35 b_{\mathrm{c}}^{2}}$

where $\alpha_{\Omega}$ is the tilt angle of the GaN layer, and $b_{\mathrm{c}}$ is the Burger's vector for screw dislocations [25, 26]. Similarly,

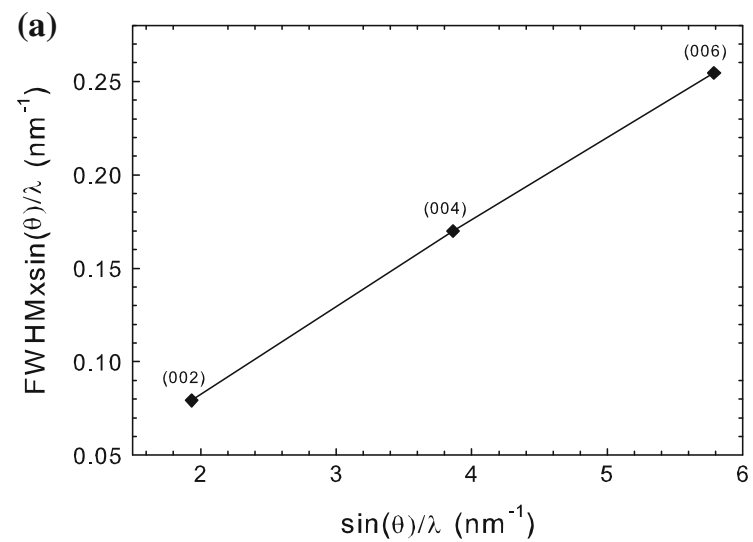

Fig. 5 The mosaic structure of a thick-GaN layer in sample B: a Williamson-Hall plot for the $(00 l) \omega$-scans, where $\ell=2,4$, and 6 . b The variation of the FWHMs for $\omega$ - and $\phi$-scans as a function of the the density of edge TDs can be obtained using the following equation:

$\rho_{\mathrm{e}}=\frac{\alpha_{\phi}^{2}}{4.35 b_{\mathrm{E}}^{2}}$

where $\alpha_{\phi}$ is the twist angle of the GaN layer, and $b_{\mathrm{E}}$ is the Burger's vector for edge dislocations [25, 26]. The screw and edge TD densities of the GaN layer were obtained as $5.4 \times 10^{7}$ and $5.0 \times 10^{9} \mathrm{~cm}^{-2}$. The screw-type dislocation density of the GaN layer is approximately two-orders of magnitude lower than the edge-type one. These results are consistent with earlier reports [13, 14]. The TDs in GaN epilayers affect the device performance due to electron scattering and current leakage paths. For instance, the dislocations can become the dominant scattering centers when the TD densities are in excess of $10^{9} \mathrm{~cm}^{-2}$ for n-type $\mathrm{GaN}$ [27].

Besides for a high density of TDs, the surface/interface roughness and cracking of the layers can severely degrade two-dimensional electron gas (2DEG) properties of GaNbased HEMTs [28]. In our previous study on surface morphology of $\mathrm{Al}_{0.3} \mathrm{Ga}_{0.7} \mathrm{~N} / \mathrm{Al}_{2} \mathrm{O}_{3}$-HEMT structure [29], we have only reported the surface properties of AlN and GaN buffer films, which are labeled as AlN buffer layer and thick-GaN layer in the present study. We have found that an AlN buffer layer have a significant impact on the surface properties of HEMT structure. Therefore, the surface characterization of the samples has been performed at the nanometric level in detail by AFM scans of 25 and $4 \mu \mathrm{m}^{2}$. Figure 6 shows AFM images with a $4 \mu \mathrm{m}^{2}$ scan area of as-grown samples. Compared to the parallel stepterraces on the AlN surface, the GaN surface exhibited randomly oriented step-terraces. In addition, most of the terraces on the GaN surface were pinned at dark spots in the AFM image. Step-flow morphology, which implies a

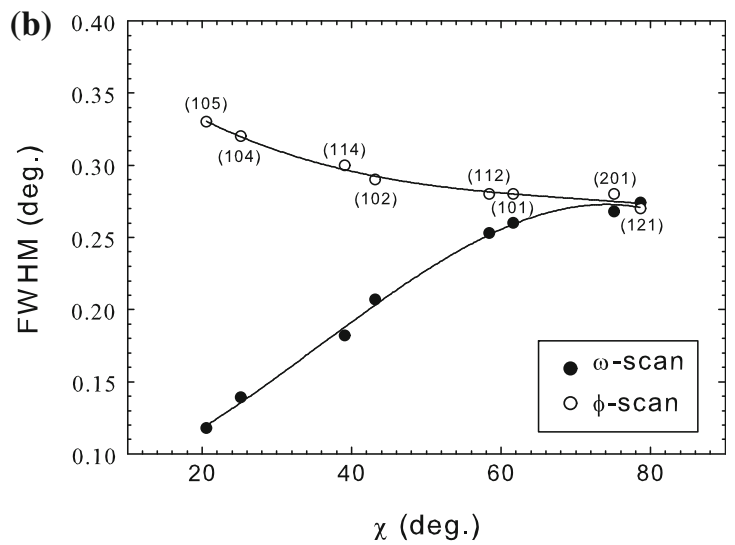

$\chi$ angle. The FWHMs of $\omega$-scans increase with the increment of the $\chi$ angle, while the FWHMs of the $\phi$-scans decrease with the increment of the $\chi$ angle 

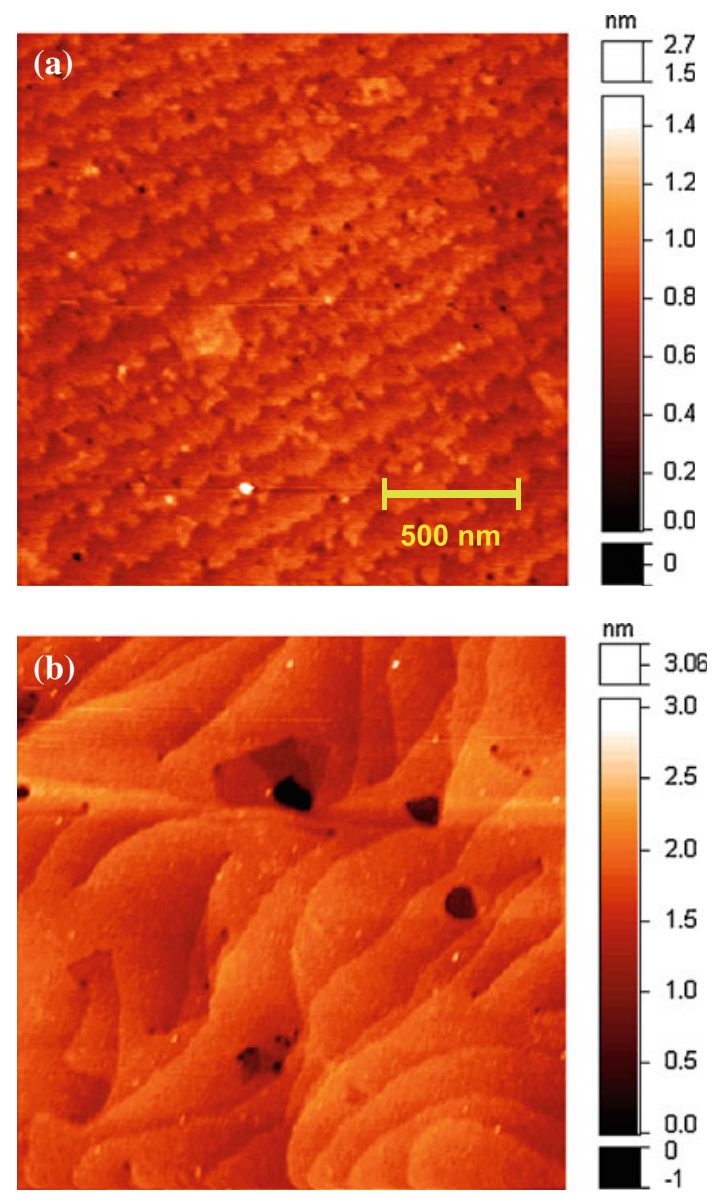

Fig. 6 AFM images with a $4 \mu \mathrm{m}^{2}$ scan area of samples a A and b B. The surface rms values of samples A and B for these scans are 0.09 and $0.25 \mathrm{~nm}$, respectively. For sample B, the density of the dark spots on the GaN surface is $6.5 \times 10^{8} \mathrm{~cm}^{-2}$

smooth surface, is important for high HEMT performance $[30,31]$. The surface rms roughnesses of samples A and B were already measured as 0.09 and $0.25 \mathrm{~nm}$ over a scan area of $4 \mu \mathrm{m}^{2}$ in Fig. 6 . Since the small probed area is more flat than the large one, the rms value obtained from the $4 \mu \mathrm{m}^{2}$ AFM scan is not an accurate representation of the sample. Hence, we measured the surface roughness from five different images, each with a $25 \mu \mathrm{m}^{2}$ scan area. The average rms values of AlN and GaN surfaces were obtained as 0.16 and $0.84 \mathrm{~nm}$, respectively. In addition, the roughness is also closely related to the lateral terrace sizes (or terrace widths) [11, 32], which are in the range of 100-200 nm for both of the samples. The measured terrace widths of the samples appear to be significant, since the nitride films with a terrace width larger than $100 \mathrm{~nm}$ exhibit smoother surfaces [32]. In this case, it can be clearly seen that the rms values and terrace widths of the samples are appropriate for smooth surfaces. Moreover, an AlN buffer layer (sample A) has an atomically flat surface. The surface quality of a layer in the heterostructure is affected from underlying layers [33]. In this case, rough surface of sample B with respect to sample A implies a defective GaN/AlN interface. Also, the surface roughness of the buffer layer affects the structural quality of a GaN epilayer. This may explain the reason of the relatively low (102) FWHM of thick-GaN layer compared to that of typically $\mathrm{GaN}$.

The dark spots at the step terminations on the GaN surface are closely related to screw-type dislocations [34]. The dark spot density of sample B was estimated as $6.5 \times 10^{8} \mathrm{~cm}^{-2}$ by the number of dark spots from the AFM scan of $4 \mu \mathrm{m}^{2}$ in Fig. 6. This result is in agreement with our previous report [11] on good quality AlGaN/GaN heterostructures grown on AlN buffer/sapphire templates by MOCVD. Also, this value is approximately one order of magnitude higher than the screw TD density calculated as $5.4 \times 10^{7} \mathrm{~cm}^{-2}$ by XRD. This result can be related to the distribution of the dark spots on the surface of the GaN layer. The black regions consisting of a few dark spots, as shown in Fig. 6 are evidence of non-uniform distribution. Additionally, since the density of the dark spots is determined by counting from over a small probed surface area, the screw TD density estimated by AFM may be relatively low or high versus what is obtained by XRD and is not an accurate representation of the sample. However, it should not be excluded that the (002) $\omega$-scans are not only broadened by mosaic tilt. On the contrary of the GaN in sample B, nearly no pinned terraces can be observed for AlN in sample A. This result shows that an AlN buffer layer has a very low density of screw-type dislocation, which is consistent with the XRD results in this study as well as the previously reported experimental results $[8,13]$.

The GaN epilayer on the $c$-plane sapphire substrate exhibits in-plane isotropic elastic properties, and its inplane deformation state can be described by one strain component [35]. The in-plane (a) and out-of-plane (c) lattice parameters of the AlN and GaN layers in the samples were calculated using Bragg law and hexagonal crystallographic formula. The strain-free (relaxed) $a$ - and $c$-lattice parameters were taken as $3.1114,4.9792 \AA$ for AlN, and 3.1897, 5.1855 $\AA$ for GaN. The obtained lattice parameters for the layers are shown in Table 1. As they are compared with the relaxed ones, it is clearly seen that samples A and B have the strained layers. The most likely reason for the large variations of the lattice parameters is the point defects that were revealed at the moment of coalescence of the nuclei such as oxygen incoming from the substrate and native defects. As is well known, the density of AlN nuclei is related to growth temperature of LT-AlN buffer layer, layer thickness, and annealing time and affects the TD density of overgrown layers. From the lattice parameters, the in-plane $\left(\varepsilon_{a}\right)$ and out-of-plane $\left(\varepsilon_{c}\right)$ strain values in perpendicular and parallel to the growth 
direction (in the $a$ - and $c$-axes) of the layers were calculated and given in Table 1. The in-plane strains for the AIN layers are tensile, but the out-of-plane strains are different types. The $\varepsilon_{\mathrm{c}}$ strain for the AlN layer in sample $\mathrm{A}$ is tensile, whereas the strain in sample $B$ is compressive type. In addition, the $\varepsilon_{\mathrm{c}}$ strain value of the AlN layer in sample $\mathrm{A}$ in comparison to sample $\mathrm{B}$ is lower, and the $\varepsilon_{\mathrm{a}}$ value is higher. The compressively strained AlN layer in sample B is probably due to the large thermal mismatch between the GaN and AlN layers.

On the other hand, the point defects in GaN films cause a crystal lattice expansion or compression because of the large difference in the covalent radii of the $\mathrm{Ga}$ and $\mathrm{N}$ atoms $\left(r_{\mathrm{Ga}}=1.26 \AA, r_{\mathrm{N}}=0.70 \AA\right)$. Harutyunyan et al. [35] reported that the $\mathrm{N}_{\mathrm{Ga}}$ substitutional-type point defects and $\mathrm{V}_{\mathrm{N}}$ and $\mathrm{V}_{\mathrm{Ga}}$ vacancies in $\mathrm{GaN}$ lead to a crystal lattice compression. As can be seen from Table 1, a thick-GaN layer in sample B exhibited compressive behavior. In this context, we suppose that the $\mathrm{N}_{\mathrm{Ga}}, \mathrm{V}_{\mathrm{N}}$, and $\mathrm{V}_{\mathrm{Ga}}$-type point defects in thick-GaN layer are dominant with respect to other types of point defects. Furthermore, strain character of the layer can be affected from the mismatched-substrate, post-growth cooling or impurities such as carbon, silicon magnesium, and oxygen incoming from the sources, doping materials or sapphire substrate. A detailed analysis on the point defects will be given in the PL part of the paper.

Figure 7 shows the room-temperature PL spectra of sample B. The spectrum includes three main transitions: a strong and sharp nearly band edge transition (BE) centered at $372 \mathrm{~nm}$, broad Gaussian-shaped blue luminescence (BL) band centered at $445 \mathrm{~nm}$, and another broad Gaussianshaped yellow luminescence (YL) band centered at $550 \mathrm{~nm}$. The impurities, generally due to unintentional doping and the native defects such as vacancies due to nonstoichiometric growth or intentional doping give rise to the main transitions. The dominant mechanism for the $\mathrm{BE}$ transition is the formation of a shallow donor due to nitrogen vacancy $\left(\mathrm{V}_{\mathrm{N}}\right)[36]$ and oxygen on the $\mathrm{N}$ sites $\left(\mathrm{O}_{\mathrm{N}}\right)$ [37] bound exciton (DBE) [38]. In general, the BL and YL bands are found in unintentionally doped material [39]. A large number of studies have been reported in the literature on the origin of $\mathrm{YL}$ and $\mathrm{BL}$ bands. The impurities and native defects are not yet well established, but the most probable impurity type is the $\mathrm{Si}$ on Ga sites $\left(\mathrm{Si}_{\mathrm{Ga}}\right)$ [40] and the most probable native defects are the gallium vacancy $\left(\mathrm{V}_{\mathrm{Ga}}\right)$ for the n-type $\mathrm{GaN}$ and the nitrogen vacancy $\left(\mathrm{V}_{\mathrm{N}}\right)$ for the p-type $\mathrm{GaN}$ [41]. The broad BL band is a characteristic of MOCVD grown undoped or unintentionally doped GaN layers [42], and is attributed to the transition from the conduction band to the deep acceptor (e-A) levels. The most probable candidates of this transition are intrinsic defects such as dislocations and low angle grain boundaries, metastable defects such as the $\mathrm{Zn}$ and $\mathrm{Mg}$ acceptor

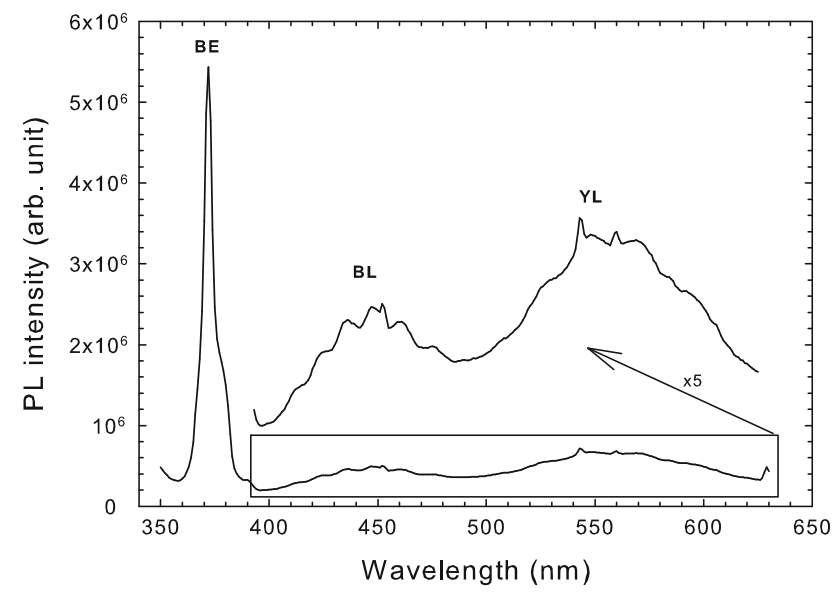

Fig. 7 Room-temperature photoluminescence spectra of sample B, which was grown using an AlN buffer

levels due to the system contamination (MO source impurities) and carbon and oxygen on the Ga sites $\left(\mathrm{C}_{\mathrm{Ga}}\right.$ and $\mathrm{O}_{\mathrm{Ga}}$ ) [42-44]. Another broad YL band is attributed to the transition of electron from the conduction band to the deep acceptor, from the shallow or deep donor to the deep or shallow acceptor, from the deep donor to the valance band, crystalline defects, and the residual impurities due to the system contamination [45-50]. Despite the main formation, the mechanism of YL is still unclear. However, the dominant mechanism is the negatively charged $\mathrm{Ga}$ vacancy, that is, deep acceptor $\left(\mathrm{C}_{\mathrm{N}}\right)$ assisted gallium vacancy $\left(\mathrm{V}_{\mathrm{Ga}} \mathrm{C}_{\mathrm{N}}\right)$ [40, 51, 52]. Strong near-band-edge emission correlates with high quality film and weak BL and YL bands correlate with metastable defects due to the system contamination and native defects due to the growth conditions, respectively. On the other hand, the stronger PL intensity can be attributed to the larger grain size, and larger grain size can be attributed to the smaller mosaic twist [53, 54]. Within this framework, the strong nearband-edge emission and weak BL and YL bands that can be clearly seen from Fig. 7 are an evidence of a large grain size and small mosaic twist, that is, the high crystalline quality of the GaN layer in sample B. The PL results are also in good agreement with the HRXRD and AFM results.

\section{Conclusion}

We investigated the structural and morphological properties of an AlN buffer layer and a thick-GaN layer grown on sapphire substrate by MOCVD. We also evaluated the optical quality of the GaN layer by using a room-temperature PL measurement. The HRXRD and PL results show that the AlN buffer and thick-GaN layers have a high crystalline quality. Furthermore, the densities of the 
screw- and edge-type dislocation for the thick-GaN layer were calculated as $5.4 \times 10^{7}$ and $5.0 \times 10^{9} \mathrm{~cm}^{-2}$ via the mosaic model. The AFM results show that the AlN buffer and the thick-GaN layers were grown as step-flow and they exhibited smooth surfaces. In addition, the dark spot density of the GaN surface was estimated as $6.5 \times 10^{8} \mathrm{~cm}^{-2}$ for the $4 \mu \mathrm{m}^{2}$ scan area.

Acknowledgements This work was supported by the Turkish State Planning Organization (Project No. 2001K120590). This work is also supported by TUBITAK under Project Nos. 105E066, 105A005, 106E198, and 106A017. One of the authors (E. O.) also acknowledges partial support from the Turkish Academy of Sciences.

\section{References}

1. Nakamura N, Furuta K, Shen XQ, Kitamura T, Nakamura K, Okumura H (2007) J Cryst Growth 301:452

2. Yu H, Caliskan D, Ozbay E (2006) J Appl Phys 100:033501

3. Lester SD, Ponce FA, Craford MG, Steigerwald DA (1995) Appl Phys Lett 66(10):1249

4. Kapolnek D, Wu XH, Heying B, Keller S, Mishra UK, DenBaars SP, Speck JS (1995) Appl Phys Lett 67(11):1541

5. Weimann NG, Eastman LF (1998) J Appl Phys 83:3656

6. Cao XA, Teetsov JA, Shahedipour-Sandvik F, Arthur SD (2004) J Cryst Growth 264:172

7. Amano H, Sawaki N, Akasaki I, Toyoda Y (1986) Appl Phys Lett 48:353

8. Bai J, Wang T, Parbrook PJ, Lee KB, Cullis AG (2005) J Cryst Growth 282:290

9. Bai J, Wang T, Comming P, Parbrook PJ, David JPR, Cullis AG (2006) J Appl Phys 99:023513

10. Poti B, Tagliente MA, Passaseo A (2006) J Non-Cryst Solids 352:2332

11. Çörekçi S, Öztürk MK, Akaoğlu B, Çakmak M, Özçelik S, Özbay E (2007) J Appl Phys 101:123502

12. Okada N, Kato N, Sato S, Sumii T, Nagai T, Fujimoto N, Imura M, Balakrishnan K, Iwaya M, Kamiyama S, Amano H, Akasaki I, Maruyama H, Takagi T, Noro T, Bandoh A (2007) J Cryst Growth 298:349

13. Ha JS, Lee HJ, Lee SW, Lee HJ, Lee SH, Goto H, Cho MW, Yao T, Hong SK, Toba R, Lee JW, Lee JY (2008) Appl Phys Lett 92:091906

14. Katagiri Y, Kishino S, Okuura K, Miyake H, Hiramatu K (2009) J Cryst Growth 311:2831

15. Jiang H, Egawa T, Hao M, Liu Y (2005) Appl Phys Lett $87: 241911$

16. Vickers ME, Kappers MJ, Datta R, McAleese C, Smeeton TM, Rayment FDG, Humpreys CJ (2005) J Phys D Appl Phys 38:A99

17. Wong YY, Chang EY, Yang TH, Chang JR, Chen YC, Ku JT, Lee CT, Chang CW (2009) J Cryst Growth 311:1487

18. Wang K, Reeber RR (2001) Appl Phys Lett 79(11):1602

19. Ahmad I, Holtz M, Faleev NN, Temkin H (2004) J Appl Phys 95(4): 1692

20. Robert R, Wang K (2001) MRS Internet J Nitride Semicond Res $6: 3$

21. Bai J, Wang T, Li HD, Jiang N, Sakai S (2001) J Cryst Growth 231:41

22. Chierchia R, Böttcher T, Heinke H, Einfeldt S, Figge S, Hommel D (2003) J Appl Phys 93(11):8918
23. Zheng XH, Chen H, Yan ZB, Han YJ, Yu HB, Li DS, Huang Q, Zhou JM (2003) J Cryst Growth 255:63

24. Yu LP, Shi JY, Wang YZ, Zhang H (2004) J Cryst Growth 268:484

25. Metzger T, Hopler R, Born E (1998) Philos Mag A 77(4):1013

26. Morse M, Wu P, Choi S, Kim TH, Brown AS, Losurdo M, Bruno G (2006) Appl Surf Sci 253:232

27. Wu XH, Fini P, Tarsa EJ, Heying B, Keller S, Mishra UK, DenBaars SP, Speck JS (1998) J Cryst Growth 189:231

28. Dabiran AM, Wowchak AM, Osinsky A, Xie J, Hertog B, Cui B, Look DC, Chow PP (2008) Appl Phys Lett 93:082111

29. Çörekçi S, Usanmaz D, Tekeli Z, Çakmak M, Özçelik S, Özbay E (2008) J Nanosci Nanotechnol 8:640

30. Stephenson GB, Eastman JA, Thompson C, Auciello O, Thompson LJ, Munkholm A, Fini P, DenBaars SP, Speck JS (1999) Appl Phys Lett 74(22):3326

31. Bai J, Dudley M, Sun WH, Wang HM, Asif Khan A (2006) Appl Phys Lett 88:051903

32. Torabi A, Ericson P, Yarranton EJ, Hooke WE (2002) J Vac Sci Technol B 20(3):1234

33. Teke A, Gökden S, Tülek R, Leach JH, Fan Q, Xie J, Özgür Ü, Morkoç H, Lisesivdin SB, Özbay E (2009) New J Phys 11:063031

34. Frank FC (1949) Discuss Faraday Soc 5:67

35. Harutyunyan VS, Aivazyan AP, Weber ER, Kim Y, Park Y, Subramanya SG (2001) J Phys D Appl Phys 34:A35

36. Pankove JI (1990) Mater Res Soc Symp Proc 162:515

37. Seifert W, Franzheld R, Butter E, Sobotta H, Riede V (1993) Cryst Res Technol 18:383

38. Dingle R, Ilegems M (1971) Solid State Commun 9:175

39. Kaufmann U, Kunzer M, Obloh H, Maier M, Manz Ch, Ramakrishnan A, Santic B (1999) Phys Rev B 59:5561

40. Reshchikova MA, Morkoç H (2005) J Appl Phys 97:061301

41. Neugebauer J, Van de Wall CG (1994) Phys Rev B 50:8067

42. Li S, Jing F, Han G, Wang L, Xiong C, Peng X, Mo H (2005) Mater Sci Eng B 122:72

43. Dhar S, Ghosh S (2002) Appl Phys Lett 80:4519

44. Kucheyev SO, Toth M, Phillips MR, Williams JS, Jagadish C (2001) Appl Phys Lett 79:2154

45. Hofmann DM, Kovalev D, Steude G, Meyer BK, Hoffmann A, Ekey L, Heitz R, Detchprohm T, Amano H, Akasaki I (1995) Phys Rev B 52:16702

46. Glaser ER, Kennedy TA, Doverspike K, Rowland LB, Gaskill DK, Freitas JA, Asif Khan M, Olson DT, Kuznia JN, Wickenden DK (1995) Phys Rev B 51:13326

47. Ogino T, Aoki M (1980) Jpn J Appl Phys 19:2395

48. Ponce FA, Bour DP, Götz W, Wright PJ (1996) Appl Phys Lett 68:57

49. Mattila T, Nieminen RM (1997) Phys Rev B 55:9571

50. Shalish I, Kronik L, Segal G, Rosenwaks Y, Shapira Y, Tisch U, Salzman J (1999) Phys Rev B 59(15):9748

51. Xing H, Keller S, Wu YF, McCarthy L, Smorchkova IP, Buttari D, Coffie R, Green DS, Parish G, Heikman S, Shen L, Zhang N, Xu JJ, Keller BP, DenBaars SP, Mishra UK (2001) J Phys Condens Matter 13:7139

52. Saarinen K, Laine $T$, Kuisma $S$, Nissilä $J$, Hautojärvi $P$, Dobrzynski L, Baranowski JM, Pakula K, Stepniewski R, Wojdak M, Wysmolek A, Suski T, Leszczynski M, Grzegory I, Porowski S (1997) Phys Rev Lett 79(16):3030

53. Wang H, Huang Y, Sun Q, Chen J, Wang LL, Zhu JJ, Zhao DG, Zhang SM, Jiang DS, Wang YT, Yang H (2006) Appl Phys Lett 89:092114

54. Kudrawiec R, Nyk M, Syperek M, Podhorodecki A, Misiewicz J, Strek W (2006) Appl Phys Lett 88:181916 